\title{
(C) OPEN ACCESS \\ Contraception and prevention of HIV transmission: a potential conflict of public health principles
}

\author{
Lisa B Haddad, ${ }^{1}$ Sean Philpott-Jones, ${ }^{2}$ Toby Schonfeld ${ }^{3}$
}

\begin{abstract}
${ }^{1}$ Assistant Professor, Department of Gynecology and Obstetrics, School of Medicine, Emory University, Atlanta, GA, USA ${ }^{2}$ Director, Center for Bioethics and Clinical Leadership, The Bioethics Program, Union Graduate College, Mount Sinai School of Medicine, New York, NY, USA

${ }^{3}$ Director, Master of Arts in Bioethics, Center for Ethics and Professor, School of Medicine, Emory University, Atlanta, GA, USA
\end{abstract}

\section{Correspondence to} Dr Lisa B Haddad, Emory University School of Medicine, Department of Gynecology and Obstetrics, 49 Jesse Hill Jr Drive, Atlanta, GA 30303, USA;

Ibhadda@emory.edu

*Details correct at time of manuscript preparation.

Received 26 November 2013 Revised 21 April 2014 Accepted 13 September 2014 Published Online First 16 October 2014

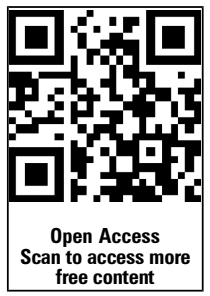

CrossMark

To cite: Haddad LB, PhilpottJones S, Schonfeld* T. J Fam Plann Reprod Health Care 2015:41:20-23.

\section{OVERVIEW}

Recent research suggests that HIV transmission and acquisition may increase with the use of some forms of hormonal contraception. Current data are not conclusive, but should it be determined that use of hormonal contraception can promote HIV spread, one consequence will be a competition between two important public health goals. The laudable goals of preventing HIV transmission and reducing rates of unintended pregnancy, particularly important in developing regions of the world like sub-Saharan Africa, will be in conflict. In this article we examine the different challenges raised by these competing goals and explore the broad range of factors at play using the six-part ethical framework developed by Baum et al. for managing ethical challenges in public health practice. We conclude that given the array of considerations required prior to restriction of hormonal contraceptive methods in resource-poor regions of the world, such restrictions should not be imposed, even if it were proven that some of these methods could exacerbate the spread of HIV among at-risk individuals.

\section{BACKGROUND}

Of the estimated 33 million people living with HIV/AIDS worldwide, 16 million are women, and infection rates are increasing in women even as the epidemic stabilises globally. ${ }^{1}$ The countries with the highest rates of HIV also have low rates of contraception use and thus high rates of unintended pregnancy. ${ }^{2}$ Improving family planning services in these countries has been a key public health goal, as preventing unintended pregnancy reduces maternal and child mortality, reduces rates of mother-tochild (vertical) transmission of HIV, reduces poverty and improves educational and gender equity.

Until recently, researchers and policymakers have often overlooked the impact of pregnancy on HIV prevention research and practice. Due to a number of biological and socioeconomic factors, current HIV prevention tools - abstinence, male or female condoms, male circumcision and monogamy - are inaccessible to many women. At-risk women are also those most likely to become pregnant, and pregnancy may increase risk for infection and transmission to their partners. ${ }^{3}$ Thus it is essential to develop new HIV prevention methods that these women can use. Unfortunately, current regulations require that women who become pregnant during trials of vaccines, microbicides or pre-exposure prophylaxis - promising new HIV prevention tools - discontinue use of the investigational agent or withdraw from the study. High rates of pregnancy among study participants also threaten the validity of HIV prevention studies by reducing their power to detect modest effects on HIV transmission.

As a result, many HIV prevention trials now require use of contraceptives as a condition of study enrolment. Not only does this ensure the scientific validity of the study, but offering contraceptives has been seen as providing benefits to at-risk women directly and to local communities indirectly. However, several studies over the past decade have questioned this wisdom.

\section{HORMONAL CONTRACEPTION AND HIV RISK}

In a recent study of serodiscordant couples it was found that women who used injectable hormonal (progestogen) contraceptives, such as depot medroxyprogesterone acetate (DMPA), were twice 
as likely to acquire HIV as those who used nonhormonal forms of birth control. ${ }^{4}$ Among HIV-positive women, injectable hormonal contraceptives also increased the transmission likelihood to an uninfected male partner. However, the validity of these data has been questioned by several studies that have conflicting findings. ${ }^{5-7}$ In addition, a very recent World Health Organization (WHO) consultation concluded that there was currently insufficient evidence to support a change in the guidelines on the use of hormonal contraceptives in sub-Saharan Africa. ${ }^{8}$ The WHO did encourage further investigation of the impact of hormonal contraception and HIV risk, as well as increased efforts to promote consistent use of condoms among DMPA users. Part of this future research programme includes the recognition that the different hormonal contraceptives may have differential impact on this potential risk, with the most concerning evidence of increased risk of acquisition of HIV notable primarily for women who use DMPA. ${ }^{7}$ For example, the risk of elevated HIV transmission from the use of combined hormonal contraceptives, progestogen-releasing intrauterine devices (IUDs) or contraceptive implants may be lower in comparison to DMPA. However, the evidence on relative contraceptive safety is both lacking and controversial. For the purpose of this article, we will deal broadly with all hormonal contraceptives since the relative influence of particular hormonal methods remains unanswered.

In view of this uncertainty, the current HIV prevention-contraception debate has created considerable tension within the public health community. It is therefore important to consider the ethical implications for current research and clinical practice proactively, in case it is proven that some hormonal contraceptives do indeed increase the risk of HIV acquisition and transmission. Should such findings be confirmed, two very important areas - family

Box 1 Baum et al.'s ${ }^{11}$ framework for addressing ethical challenges in public health practice

Six considerations in addressing ethical challenges in public health practice:

- Determine population-level utility of the proposed action

- Demonstrate evidence of need and effectiveness of the action

- Establish fairness of goals and proposed implementation strategies

- Demonstrate accountability

- Assess expected efficiencies and costs associated with the proposed action

- Consider political feasibility and community acceptance planning and HIV prevention - could become competing public health goals.

\section{COMPETING PUBLIC HEALTH CONCERNS}

Several authors have identified ways to assess and address ethical challenges in public health practice. For example, the Principles of the Ethical Practice of Public Health, published by the USA Public Health Leadership Society, serve as a code of ethics (of sorts) for public health practitioners. ${ }^{9}$ Similarly, Childress and his colleagues have identified a set of general moral considerations that are often in play with regard to public health practice. ${ }^{10}$ However, none of these strategies describe conditions or provide solutions for situations where two public health principles are in direct competition with each other, as is the case with the HIV prevention versus contraception conundrum.

\section{BAUM ET AL.'S FRAMEWORK FOR ETHICAL CHALLENGES}

Baum et al. ${ }^{11}$ developed a framework for managing ethical challenges in public health practice. We believe that applying this framework provides a productive way to reconcile the potentially competing goals of family planning and HIV prevention, especially given the interconnections between the ethical, political and contextual features of these two public health issues. Baum and her colleagues offer six considerations for any proposed action in public health. These are listed in Box 1. We apply them below to the issues of HIV prevention and prevention of unplanned pregnancy in high-risk groups, focusing specifically on the implications of the finding that certain hormonal contraceptives might increase rates of HIV transmission.

- Determine population-level utility of the proposed action - Reducing use of hormonal contraceptive methods in this population could decrease rates of transmission of HIV. Given that an estimated 14 million unintended pregnancies occur annually in this population, however, the utility of pregnancy prevention for women living with HIV/AIDS is dually critical: to prevent the unnecessary morbidity and mortality associated with pregnancy, and to prevent vertical transmission to infants of HIV-infected mothers. ${ }^{2}$ Given the poor utilisation of barrier contraceptive methods in high-risk communities, appeal to population-level utility may call into question the wisdom of removing the one practical and highly effective pregnancy prevention system in the region.

- Demonstrate evidence of need and effectiveness of the action - Sub-Saharan Africa has the lowest contraceptive use in the developing world, as well as the largest gap between knowledge regarding contraception and current use. ${ }^{12}$ This is attributable in part to cultural pressures and 
issues of access. Nevertheless, more than 7000 new HIV infections are documented globally each day among those being tested for HIV, probably an underestimate of the true incidence rate. ${ }^{13}$ These data suggest that there is a clear need for effective HIV prevention strategies, but also for increased sensitivity to contextual considerations when balancing the HIV prevention goal with that of pregnancy prevention.

- Establish fairness of goals and proposed implementation strategies - There must be a just sharing of benefits and burdens of any proposed policy. While reducing HIV transmission risk benefits all, reducing the availability of effective contraception affects women disproportionately. Women shoulder the burden of pregnancy and delivery while also performing the vast majority of childcare, which reduces their other opportunities in society. Therefore, any proposed policy must consider how the implementation of an action will affect sectors of the population differently.

- Demonstrate accountability - Transparency is a necessary virtue in public health, particularly in situations where there are clearly competing public health principles. Clarity about the goals of the proposed action is essential, as is ensuring that those policies are based on sound, credible and reproducible scientific data. In cases where individual liberty may be compromised or where certain sectors of the population bear disproportionate burdens, transparency in the values that justify the proposed actions will demonstrate the credibility of the decision-makers, who have ultimate accountability for their dissemination and implementation.

- Assess expected efficiencies and costs associated with the proposed action - The costs of both pregnancy prevention and reduction of HIV transmission - as well as costs, financial and otherwise, of not achieving those goals - must be considered. Long-acting reversible contraceptive methods are the most cost-effective methods, yet there is poor uptake in sub-Saharan Africa. Wide-scale HIV treatment is a cost-saving strategy that can significantly reduce morbidity and mortality among people living with HIV/AIDS, as well as reducing the transmission of HIV to their partners and children, but the initiation and maintenance of antiretroviral therapy has been inconsistent in resource-poor areas.

- Consider political feasibility and community acceptance - The political feasibility of proposed options may differ from country to country as policymakers weigh the risks and benefits of using hormonal contraceptives on rates of HIV transmission, unintended pregnancy, and maternal mortality. As a result, hormonal contraceptive methods that may be deemed suitable for one country may not acceptable for another. In addition, gender-related power structures pose barriers to family planning in the sub-Saharan Africa region. It is typically the woman's responsibility to seek family planning services, but men usually control sexual and familial economic decisionmaking. Many women also rely on their spouses for income. This complicates access to family planning services in a number of ways: women face financial barriers to purchasing contraception, cultural barriers to asking partners to use condoms, and social barriers to child number and spacing. The copper IUD, a non-hormonal contraceptive option, is a discreet alternative to hormonal contraception, but currently uptake and utilisation remain poor in these high-risk communities. Therefore, hormonal contraceptives, which require nothing from the male partner and that have a lower risk of discovery should covert use be necessary, are likely to be superior to the alternative options.

\section{CONCLUSIONS}

Applying the Baum framework to the ethical challenges presented by the competing public health goals of family planning and prevention of HIV transmission suggests that effective management of that potential conflict requires the consideration of a broad array of factors. Policymakers should consider the disproportionate harm to women that may follow the reduction in availability of effective and practicable pregnancy prevention measures. Without careful attention to education, increasing access to services, and sensitivity to context when formulating new clinical and research strategies, any decision to reduce access to hormonal contraception for women at risk of acquiring HIV is premature and likely to cause greater harm than good. Individual-level choice regarding contraception should remain with the individual woman in consultation with her health care provider. That said, in certain regions with higher HIV prevalence, the balance might tip such that use of certain contraceptives might be less desirable. ${ }^{14}$ Finally, increased condom use would of course mitigate both public health issues and resolve the 'competition' between HIV prevention and family planning programmes. Dual protection with consistent and correct use of condoms in addition to a hormonal contraception is an imperative strategy that needs further promotion. ${ }^{15}$ However, until cultural, social and religious contexts alter sufficiently to facilitate widespread use of condoms, this solution will remain ideal rather than real.

Competing interests None.

Provenance and peer review Not commissioned; externally peer reviewed. 
Open Access This is an Open Access article distributed in accordance with the Creative Commons Attribution Non Commercial (CC BY-NC 4.0) license, which permits others to distribute, remix, adapt, build upon this work noncommercially, and license their derivative works on different terms, provided the original work is properly cited and the use is non-commercial. See: http://creativecommons.org/licenses/by$\mathrm{nc} / 4.0 /$

\section{REFERENCES}

1 Joint United Nations Programme on HIV/AIDS (UNAIDS). Global Report. UNAIDS Report on the Global AIDS Epidemic. Geneva, Switzerland: UNAIDS, 2010.

2 Hubacher D, Mavranezouli I, McGinn E. Unintended pregnancy in sub-Saharan Africa: magnitude of the problem and potential role of contraceptive implants to alleviate it. Contraception 2008;78:73-78.

3 Mugo NR, Heffron R, Donnell D, et al. Partners in Prevention HSV/HIV Transmission Study Team. Increased risk of HIV-1 transmission in pregnancy: a prospective study among African HIV-1-serodiscordant couples. AIDS 2011;25:1887-1895.

4 Heffron R, Donnell D, Rees H, et al; for the Partners in Prevention HSV/HIV Transmission Study Team. Use of hormonal contraceptives and risk of HIV-1 transmission: a prospective cohort study. Lancet Infect Dis 2012;12:19-26.

5 Morrison CS, Nanda K. Hormonal contraception and HIV: an unanswered question. Lancet Infect Dis 2012;12:2-3.

6 Polis CB, Phillips SJ, Curtis KM. Hormonal contraceptive use and female-to-male HIV transmission: a systematic review of the epidemiologic evidence. AIDS 2013;27:493-505.
7 Polis CB, Phillips SJ, Curtis KM, et al. Hormonal contraceptive methods and risk of HIV acquisition in women: a systematic review of epidemiological evidence. Contraception 2014;90:360-390.

8 World Health Organization. Hormonal Contraceptive Methods for Women at High Risk and Living with HIV. 2014 Guidance Statement. 2014. http://apps.who.int/iris/bitstream/10665/ 128537/1/WHO_RHR_14.24_eng.pdf?ua=1 [accessed 14 September 2014].

9 Public Health Leadership Society. Principles of the Ethical Practice of Public Health (Version 2.2). 2002. http://nnphi.org/ uploads/media_items/principles-of-the-ethical-practice-of-publichealth-brochure.original.pdf [accessed 21 February 2012].

10 Childress JF, Faden RR, Gaare RD, et al. Public health ethics: mapping the terrain. J Law Med Ethics 2002;30:170-178.

11 Baum NM, Gollust SE, Goold SD, et al. Looking ahead: addressing ethical challenges in public health practice. J Law Med Ethics 2007;35:657-667.

12 Khan S, Mishra V, Arnold F, et al. Contraceptive trends in developing countries (DHS Comparative Reports 16). 2007. http://dhsprogram.com/pubs/pdf/CR16/CR16.pdf [accessed 14 September 2014].

13 Cohen MS, Gay CL, Busch MP, et al. The detection of acute HIV infection. J Infect Dis 2010;202(Suppl. 2):S270-S277.

14 Rodriguez MI, Reeves MF, Caughey AB. Evaluating the competing risks of HIV acquisition and maternal mortality in Africa: a decision analysis. BJOG 2012;119:1067-1073.

15 Edouard L. The renaissance of barrier methods. J Fam Plann Reprod Health Care 2012;38:131-133. 